\title{
Main Bronchus
}

National Cancer Institute

\section{Source}

National Cancer Institute. Main Bronchus. NCI Thesaurus. Code C12284.

The left and right main bronchi considered as a group. 